\title{
Estratégias para a segurança do paciente no processo de uso de medicamentos após alta hospitalar
}

\section{| ${ }^{1}$ Liete de Fátima Gouveia Marques, ${ }^{2}$ Nicolina Silvana Romano-Lieber |}

Resumo: O atual movimento mundial para segurança do paciente reforça a necessidade de desenvolver prevenção quaternária - visando proteger os pacientes do excessivo intervencionismo diagnóstico e terapêutico e minimizar o risco de iatrogenias para evitar danos ao paciente e gastos desnecessários ao sistema de saúde. Em situaçôes de transição do cuidado, como alta hospitalar, a ocorrência de eventos adversos relacionados ao uso de medicamentos pode resultar em readmissão hospitalar ou necessidade de atendimento ambulatorial ou em serviços de urgência. Este artigo tem por objetivo discutir o gerenciamento da segurança do paciente no processo de uso de medicamentos após alta hospitalar. Com base em pesquisa bibliográfica, realizou-se abordagem sobre estratégias utilizadas por equipe hospitalares, tais como reconciliação medicamentosa, orientação ao paciente e/ou cuidador, comunicação por meio de resumo de alta, e seguimento domiciliar, além da necessidade de atuação interdisciplinar e interinstitucional para contribuir com a integralidade do cuidado. Concluise que o desenvolvimento de atividades de cuidado ao paciente em alta, pela equipe hospitalar, e a existência de efetiva articulação entre o hospital e demais serviços de saúde podem representar importantes aliados neste grande desafio para a saúde pública, que é a segurança do paciente no processo de uso de medicamentos após alta hospitalar.

> Palavras-chave: medicamento; eventos adversos; hospital; segurança do paciente; Saúde Pública.

\author{
${ }^{1}$ Faculdade de Saúde Pública \\ da Universidade de São Paulo. \\ São Paulo, Brasil. Endereço \\ eletrônico: liete.marques@ \\ hotmail.com \\ 2 Departamento de Prática de \\ Saúde Pública, Faculdade de \\ Saúde Pública - USP. São Paulo, \\ Brasil. Endereço eletrônico: \\ nicolina@usp.br
}

Recebido em: 04/06/2013 Aprovado em: 02/05/2014 


\section{Introdução}

A segurança do paciente, entendida como "redução do risco, a um mínimo aceitável, de danos desnecessários associados ao atendimento em saúde" (RUNCIMAN et al., 2009, p. 19), é tema de interesse dos próprios pacientes e seus cuidadores, profissionais e instituições de saúde, instituições de ensino e pesquisa, e organizaçôes governamentais e não-governamentais. A Aliança Mundial para a Segurança do Paciente, ${ }^{1}$ lançada em 2004 pela Organização Mundial da Saúde com o objetivo de facilitar o desenvolvimento de políticas e práticas para a segurança do paciente, recomendou aos países atenção ao tema. Recentemente, importante passo nessa direção foi dado pelo Ministério da Saúde brasileiro, ao instituir o Programa Nacional de Segurança do Paciente (BRASIL, 2013).

A preocupação com a segurança do paciente tem reforçado a necessidade de desenvolver a prevenção quaternária, que visa proteger os pacientes do excessivo intervencionismo diagnóstico e terapêutico e minimizar o risco de iatrogenias (NORMAN; TESSER, 2009). A publicação de vários estudos, nas últimas décadas, tem auxiliado a compreender a dimensão do problema e importância do manejo dos riscos associados ao uso de medicamentos. Em revisão sistemática realizada por Vries e colaboradores (2008), a incidência de eventos adversos ${ }^{2}$ (EAs) entre os pacientes foi de 3,2\% a 16,6\%, sendo que o percentual médio de EAs considerados evitáveis foi de 43,5\%. Cerca de 80\% dos EAs foram detectados no hospital e $15 \%$ fora dele, antes da admissão ou após alta hospitalar. Com relação aos desfechos, $56,3 \%$ dos EAs foram considerados leves, 7\% resultaram em incapacidade permanente, e 7,4\% dos eventos causaram a morte dos pacientes. Os eventos eram, principalmente, relacionados a procedimentos cirúrgicos (40\%) ou medicamentos (15\%).

Mendes e colaboradores (2005), em revisão sistemática de estudos de avaliação da ocorrência de eventos adversos em hospitais de sete países, demonstraram que a incidência de EAs variou de 2,9 a 16,6 por 100 pacientes. Do total de eventos adversos, $38 \%$ estavam relacionados a procedimentos cirúrgicos e 19\%, a medicamentos. Em estudo realizado em três hospitais brasileiros, a incidência de pacientes com EAs foi de 7,6\%, sendo que 35,2\% dos eventos estavam relacionados a procedimentos cirúrgicos e 5,6\% a medicamentos. Embora a taxa de incidência tenha sido considerada similar à 
encontrada em outros estudos, a proporção de EAs evitáveis $(66,7 \%)$ foi mais elevada no estudo brasileiro (MENDES et al., 2009).

Em revisão realizada por Cano e Rozenfeld (2009), a proporção de pacientes com eventos adversos relacionados a medicamentos ${ }^{3}$ (EAMs) durante a hospitalização variou de $1,6 \%$ a $41,4 \%$, e as taxas variaram de 1,7 a 51,5 eventos por 100 hospitalizações e de 4,4 a 7,0 eventos por 100 pacientes-dia. Houve predominância de eventos classificados como leves ou moderados (mais de $80 \%$ do total), e $14,8 \%$ a $59 \%$ dos eventos foram considerados evitáveis. Revisão sistemática da literatura realizada para estimar a ocorrência de EAMs em atendimento ambulatorial demonstrou variação da taxa de prevalência conforme a faixa etária, sendo de 2,45\% para crianças, 5,27\% para adultos, e $16,1 \%$ para pacientes idosos (TACHÈ et al., 2011).

Em revisão sistemática realizada por Kongkaew e colaboradores (2008), a taxa de admissão hospitalar devido à reação adversa a medicamentos ${ }^{4}$ (RAM) variou conforme faixa etária, sendo de $4,1 \%$ para crianças, $6,3 \%$ para adultos e $10,7 \%$ para idosos. RAMs foram responsáveis ou tiveram participação em 6,6\% (9/135) das internações analisadas em hospital universitário do interior de São Paulo (PFAFFENBACH et al., 2002). E no estudo realizado por Varallo (2010), também no Estado de São Paulo, a prevalência de internações hospitalares por RAMs foi de 46,4\% (115/248). Nesse estudo, a maioria das reaçôes adversas foi classificada como possível $(66,3 \%)$ ou provável (30,3\%).

Em estudo desenvolvido em quatro hospitais de ensino de Salvador, a taxa de admissão hospitalar devida a RAMs foi de 0,56\% (212/37658). A maioria dos pacientes teve recuperação sem sequelas $(94,3 \%)$, e houve um caso fatal devido a acidente vascular cerebral causado pelo uso de varfarina (NOBLAT et al., 2011). Outro estudo, realizado em serviço de emergência no sul do Brasil (ANDREAZZA et al., 2011), verificou que, de 335 pacientes, 106 (31,6\%) foram atendidos devido a problemas relacionados com medicamentos. Os problemas mais comuns foram RAM $(28,5 \%)$ e inefetividade terapêutica devido a doses inadequadas (17,9\%).

Em estudo sobre agravos provocados por medicamentos em pacientes internados em hospitais do Sistema Único de Saúde, no Rio de Janeiro, entre 1999 e 2002, foram identificados 3.421 casos, equivalentes a 1,8 casos/1.000 
internaçôes, sendo a maioria de efeitos adversos, ${ }^{5}$ além dos casos de intoxicações (ROZENFELD, 2007).

$\mathrm{Na}$ Inglaterra, a estimativa do custo anual de admissões hospitalares decorrentes de reações adversas a medicamentos foi de $£ 466$ milhões (PIRMOHAMED et al., 2004). E segundo dados do relatório do Instituto de Medicina norte-americano de 1999 (KOHN et al., 2000), nos EUA, anualmente, sete mil mortes seriam resultantes de $\operatorname{erros}^{6}$ relacionados ao uso de medicamentos, em nível hospitalar ou ambulatorial, e os eventos adversos evitáveis relacionados a medicamentos aumentariam os custos hospitalares em dois bilhões de dólares.

$\mathrm{O}$ panorama apresentado demonstra que a ocorrência de eventos adversos relacionados a medicamentos representa uma importante causa de morbidade e mortalidade, resultando em sofrimento e aumento dos custos do atendimento em serviços de saúde, em nível mundial. Os dados reforçam a relevância da utilização de estratégias para evitar a ocorrência de eventos adversos, tanto pelo impacto social como econômico, permitindo melhor utilização dos recursos financeiros destinados ao atendimento à saúde da população.

\section{Alta hospitalar e os riscos associados ao processo de uso de medicamentos}

A questão da segurança do paciente assume particular importância em situações de transição do cuidado, como a alta hospitalar. Estima-se que 11\% a 23\% dos pacientes apresentam eventos adversos relacionados a medicamentos após alta hospitalar, sendo que $6 \%$ a $27 \%$ dos eventos poderiam ter sido evitados, e $6 \%$ a 33\% poderiam ter severidade e duração reduzidas (FOSTER et al., 2003, 2004, 2005). Além disso, estima-se que $6 \%$ a $12 \%$ dos eventos adversos relacionados a medicamentos que ocorrem após alta resultam em atendimento em serviços de urgência e 5\% em readmissão hospitalar (SCHINIPPER et al., 2006).

Os pacientes podem estar especialmente vulneráveis a danos imediatamente após alta hospitalar, por várias razões: falta de preparo para o autocuidado; falta ou inadequação de orientação; falta de acesso a profissionais de saúde que tenham conhecimento de seu plano de cuidado, quando as dúvidas surgem; descontinuidade do cuidado na interface do atendimento hospitalar e ambulatorial; transferência incorreta ou incompleta de informações, além de ausência de sistema integrado 
de informações nos diferentes serviços de atendimento à saúde do paciente (BARNSTEINER, 2005; COLEMAN et al., 2006; BAYLEY et al., 2007).

A falta de informaçôes acuradas e completas sobre quais medicamentos o paciente estava utilizando antes da internação pode levar à interrupção ou inadequação da terapia medicamentosa durante a hospitalização, ou resultar em falha na detecção de problemas relacionados a medicamentos como causa da admissão hospitalar, afetando adversamente a segurança do paciente. Na alta hospitalar, a manutenção destes erros pode resultar na prescrição de terapia farmacológica inapropriada, seja pela omissão de medicamentos anteriormente utilizados pelo paciente, ou devido à duplicação de medicamentos, presença de interações medicamentosas, manutenção de medicamentos desnecessários, desencadeando eventos adversos que podem resultar em sofrimento e custos adicionais no atendimento (CORNISH et al., 2005).

Muitos pacientes relatam que, durante hospitalização, não tiveram oportunidade de fazer perguntas sobre seus medicamentos para qualquer membro da equipe de saúde (HOLLOWAY, 1996). Frequentemente, os pacientes recebem alta hospitalar com informação inadequada ou insuficiente sobre seus medicamentos ou mesmo não recebem qualquer orientação sobre seu tratamento (CALABRESE et al., 2003; KERZMAN et al., 2005). O conhecimento insuficiente sobre seus problemas de saúde e medicamentos é uma das principais causas da falta de adesão do paciente ao seu regime farmacoterapêutico e plano de monitorização, que pode levar ao comprometimento dos resultados do tratamento, deterioração do estado de saúde do paciente, além de aumento da utilização e dos custos do atendimento em saúde (ASHP, 1997; SILVA et al., 2000; KERZMAN et al., 2005).

Em geral, os hospitais não oferecem seguimento do paciente imediatamente após alta hospitalar, em seu domicílio, até seu retorno ao acompanhamento ambulatorial. A falta de seguimento adequado do paciente é uma das causas do comprometimento da segurança dos pacientes durante o período vulnerável da transição de atendimento (COLEMAN et al., 2006).

O seguimento do paciente em outros serviços de saúde pode ser comprometido por falhas na comunicação e transferência de informações entre os profissionais envolvidos no cuidado do paciente, devido à falta de articulação das ações e serviços 
em todos os níveis de complexidade do atendimento (GLINTBORG et al., 2007). Até pouco tempo, apesar de comuns e de grandes consequências para o paciente, os erros na troca de informações e na transferência de pacientes despertavam pouca atenção - em parte porque ocorrem nas interfaces das relações profissionais (WACHTER, 2010). Atualmente, porém, tem sido enfatizada a necessidade de transferência de informações acuradas, completas e dentro de período adequado para a continuidade do cuidado ao paciente (CUA; KRIPALANI, 2008).

Estudos demonstram que algumas atividades desenvolvidas durante a hospitalização e após a alta hospitalar podem reduzir as discrepâncias entre regimes farmacoterapêuticos pré e pós-hospitalização, melhorar a adesão ao tratamento, reduzir a taxa de eventos adversos relacionados a medicamentos, além de reduzir a necessidade de novos atendimentos em serviços de saúde, tanto em emergência como readmissões hospitalares (NAYLOR et al., 1999; HUANG; LIANG, 2005; KABOLI et al., 2006; SCHNIPPER et al., 2006; COLEMAN et al., 2006).

\section{Estratégias para a segurança do paciente no processo de uso de medicamentos após alta hospitalar}

Várias estratégias têm sido divulgadas para promover efetiva transição do cuidado na alta hospitalar, e há evidências de que algumas intervenções podem ter impacto positivo sobre a segurança do paciente, especialmente aquelas com componentes educacionais e aquelas que combinam intervenções pré e pós-alta (MISTIAEN et al., 2007).

As principais estratégias direcionadas à prevenção e manejo da ocorrência de eventos adversos relacionados ao uso de medicamentos são: reconciliação medicamentosa, orientação ao paciente e/ou cuidador, seguimento domiciliar do paciente após alta hospitalar, comunicação efetiva entre hospital e demais serviços de saúde, e articulação do cuidado entre o hospital e os demais serviços de atendimento à saúde do paciente.

\section{Reconciliação medicamentosa}

Areconciliação medicamentosaéum dos procedimentosoperacionais recomendados pelo High 5's Project - projeto internacional lançado pela Organização Mundial da Saúde, em 2006, com a missão de facilitar a implantação e avaliação de soluções padronizadas para a segurança do paciente (WILLIAMS, 2012). 
Reconciliação medicamentosa é a avaliação global do regime de medicação do pa-

ciente, sempre que ocorre alteração no tratamento, em um esforço para evitar erros de medicação tais como, omissões, duplicações, erros de dose, ou interaçôes medicamentosas, assim como para observar padróes de adesão ao tratamento (CHEN; BURNS, 2007, p. 4).

A reconciliação medicamentosa permite detectar, interceptar e corrigir discrepâncias entre o tratamento prévio e o tratamento proposto, suspender medicamentos desnecessários, e revisar a segurança e adequabilidade do regime farmacoterapêutico (KRIPALANI, 2007a). Deve ser realizada reconciliação sempre que novos medicamentos são prescritos ou são refeitas prescrições já existentes, incluindo mudanças de setor, serviço, médico ou nível de atendimento, e o processo compreende as seguintes etapas: desenvolvimento de uma lista dos medicamentos em uso, como resultado da obtenção da história medicamentosa; desenvolvimento de uma lista de medicamentos a serem prescritos; comparação entre as duas listas; tomada de decisão baseada na comparação; além de comunicação da nova lista ao paciente, ao seu cuidador, e ao médico que dará continuidade ao atendimento do paciente fora do hospital (BARNSTEINER, 2008; MORIEL et al., 2008).

Schnipper e colaboradores (2006) e Vira e colaboradores (2006) verificaram que $41 \%$ a $49 \%$ dos pacientes apresentaram discrepâncias entre o tratamento prescrito na alta e o tratamento utilizado antes da hospitalização. O principal tipo de discrepância detectado nesses trabalhos foi a omissão de medicamentos anteriormente utilizados pelos pacientes (42\% a 60\% das discrepâncias), além de diferenças na dose, frequência, horário, via e forma de administração dos medicamentos. No estudo realizado por Boockvar e colaboradores (2004), $57 \%$ das alterações nos regimes farmacoterapêuticos no momento da alta se deveram à descontinuidade do medicamento, além de alteração na dose (21\%) e substituições de medicamentos (7\%).

Vários estudos avaliaram o potencial de dano das discrepâncias detectadas: $59 \%$ das discrepâncias poderiam resultar em dano se o erro não fosse interceptado e continuasse após alta hospitalar (GLEASON et al., 2004); 23\% das discrepâncias foram consideradas graves, com risco de hospitalização ou alteração persistente da função (PIPPINS et al., 2008); e mais de um terço das discrepâncias tinham potencial de causar dano moderado $(32,9 \%)$ ou severo (5,7\%), em estudo realizado com pacientes idosos (CORNISH et al., 2005). 
Revisão sistemática sobre reconciliação medicamentosa em hospitais, envolvendo 26 estudos realizados em dez países, demonstrou consistente redução nas discrepâncias de medicamentos (17 de 17 estudos), nos potenciais eventos adversos relacionados a medicamentos ( 5 de 6 estudos), e em eventos adversos relacionados a medicamentos ( 2 de 2 estudos). Dois entre oito estudos demonstraram redução na utilização de serviços de saúde após alta hospitalar. Segundo os autores, as evidências disponíveis apoiam intervenções que utilizam de forma intensiva a equipe da farmácia (farmacêuticos, residentes ou técnicos de farmácia). Os elementos comuns das intervenções que obtiveram sucesso foram: foco em subgrupos de alto risco, realização de intervenção em uma população definida (por exemplo, em unidade de cirurgia eletiva) e evidência de apoio institucional (MUELLER et al., 2012).

\section{Orientação ao paciente e/ou cuidador}

A orientação ao paciente tem por objetivo ajudá-lo a obter os maiores benefícios com o uso de seus medicamentos; auxiliar na resolução de problemas existentes; prevenir a ocorrência de problemas futuros, e desenvolver a capacidade do indivíduo em lidar com problemas relacionados ao uso de medicamentos que possam vir a ocorrer em seu domicílio (RANTUCCI, 2007).

Cua e Kripalani (2008) recomendam que, no momento da alta, sejam destacadas as alterações no tratamento que o paciente fazia antes da hospitalização, detectadas no processo de reconciliação medicamentosa na alta hospitalar, para que não haja dúvidas quanto ao tratamento que deverá realizar no domicílio, especialmente se deve ou não continuar a fazer uso de seus medicamentos. A orientação adequada, após reconciliação, possibilita evitar erros de medicação, especialmente por meio da abordagem cuidadosa quanto à identificação dos medicamentos prescritos na alta e dos medicamentos em uso anterior à hospitalização, em função da possibilidade de estoque remanescente no domicílio do paciente. Assim, recomenda-se atenção especial em duas situações específicas:

- Medicamentos de uso contínuo podem ter sido substituídos por equivalente terapêutico durante a hospitalização, em função de adaptação ao formulário terapêutico do hospital (lista de medicamentos padronizados para uso na instituição). Na alta, deve ser avaliada a possibilidade de retorno 
ao medicamento que o paciente estava utilizando antes da hospitalização

(BOOCKVAR et al., 2004; CUA; KRIPALANI, 2008), e proceder a orientação adequada ao paciente.

- O nome do medicamento na receita de alta pode confundir o paciente, podendo levar ao uso em duplicidade ou omissão do medicamento, se for prescrito pelo nome comercial e o paciente já estiver utilizando um produto genérico do mesmo medicamento, ou vice-versa (CUA; KRIPALANI, 2008). Apesar de os medicamentos de referência e produtos genéricos apresentarem os mesmos fármacos, eles podem parecer completamente diferentes para o paciente, o qual, sem orientação, pode fazer uso inadequado dos medicamentos. Além disso, os produtos disponíveis no comércio podem ter diferenças na apresentação, por exemplo, na concentração do fármaco, propiciando erros de dose (STUFFKEN; EGBERTS, 2004).

Ao final da orientação, deve ser fornecida cópia do resultado da reconciliação, com informações claras sobre quais medicamentos foram excluídos, incluídos ou tiveram o esquema posológico alterado, incluindo as justificativas.

Estudo realizado em hospital universitário norte-americano com cerca de 200 pacientes, com média de idade de 60 anos, revelou que 96\% dos pacientes tinham apresentado, na alta hospitalar, pelo menos uma alteração no regime farmacoterapêutico, com relação ao tratamento utilizado antes da hospitalização. Entretanto, apenas $44 \%$ foram alertados explicitamente sobre novos medicamentos prescritos ou alterações de doses, e 12\% receberam instruçôes por escrito para parar de utilizar medicamentos que faziam uso antes da hospitalização e que foram suspensos (UNROE et al., 2010).

Além da orientação de alta, recomenda-se que, durante o período de hospitalização, sejam realizadas sessões de orientação abordando informações sobre cada medicamento que deverá ser utilizado após alta, incluindo tratamentos prévios com medicamentos de uso contínuo.

\section{Comunicação entre hospital e demais serviços de saúde}

Informaçóes sobre o plano de alta relevantes à continuidade do tratamento devem ser fornecidas ao paciente e ao profissional de saúde que realizará o seguimento após alta. $\mathrm{O}$ resumo de alta é um importante instrumento para 
estreitar a comunicação entre o hospital e o cuidado fornecido em serviços de atenção primária, e deve conter as seguintes informações: motivo da admissão hospitalar e intervenções realizadas durante a hospitalização; tratamento proposto (nome, dose, frequência e via de administração, duração planejada de uso e razão de uso de cada medicamento), orientaçôes sobre monitorização (o que fazer, quando fazer e o que esperar dos procedimentos de monitorização), além das justificativas de exclusões, inclusões e substituições de medicamentos e/ou alterações de doses, resultantes da reconciliação; esclarecimentos sobre circunstâncias nas quais o paciente deve contatar um profissional, para quem ou onde telefonar para esclarecer dúvidas (RATLIFF, 1981; ROMANO, 1982; MAMON et al.,1992; KRIPALANI et al., 2007a).

Em revisão sistemática da literatura, Kripalani e colaboradores (2007b) demonstraram que deficiências na comunicação e na transferência de informações na alta hospitalar são comuns, sendo frequente a falta de informações importantes, no resumo de alta, tais como medicamentos receitados na alta ( $2 \%$ a $40 \%$ ) e planos de seguimento (2\% a 43\%). Bergkvist e colaboradores (2009) verificaram que alteraçóes realizadas no resumo de alta, visando melhorar a qualidade do documento, reduziram em $45 \%$ os erros de medicação de pacientes após alta hospitalar. Estudo realizado por Van Walraven e colaboradores (2002) demonstrou redução do risco de readmissão hospitalar para pacientes idosos cujos médicos tiveram acesso ao resumo de alta na consulta de seguimento após alta hospitalar.

\section{Seguimento domiciliar do paciente após alta hospitalar}

O hospital pode oferecer seguimento ao paciente por um período de tempo após alta hospitalar, especialmente durante os primeiros dias da transição do atendimento. $\mathrm{O}$ contato realizado após a alta transmite segurança ao paciente e seus familiares de que suas dúvidas e necessidades, durante esse período vulnerável, serão conhecidas e resolvidas pela equipe de saúde (COLEMAN et al., 2006).

Telefonar ao paciente poucos dias após a alta oferece a ele a oportunidade de esclarecer dúvidas sobre o regime farmacoterapêutico, relatar novos sintomas que podem estar relacionados ao uso de medicamentos, ou ainda relatar dificuldades na aquisição de medicamentos (KRIPALANI et al., 2007a). Outro recurso é a visita domiciliar pós-alta, que possibilita esclarecer dúvidas, observar como os 
medicamentos estão sendo utilizados e armazenados, descartar medicamentos antigos (CUA; KRIPALANI, 2008), reforçar orientações, monitorar progresso e aparecimento de eventos adversos, realizar ajustes no regime farmacoterapêutico, e encaminhar o paciente a serviços necessários antes que ocorra piora do quadro (NAYLOR et al., 1999).

Oliveira e Reis (2012) destacam a importância da atuação articulada entre os vários profissionais de saúde (médicos, enfermeiros, assistentes sociais, farmacêuticos e psicólogos) junto ao paciente e seus familiares, para a prevenção quaternária, especialmente no caso dos serviços domiciliares. Para paciente de alto risco, recomenda-se que seja realizada uma visita de seguimento após 48 horas da alta; para paciente de risco moderado, seguimento por telefone dentro de 48 horas e visita domiciliar dentro de cinco dias (BISOGNANO; BOUTWELL, 2009).

Schnipper e colaboradores (2006), em seguimento realizado de três a cinco dias após alta de 79 pacientes, verificaram que 12 pacientes não estavam utilizando um dos medicamentos da receita de alta, 11 utilizavam dose ou frequência diferente da prescrita e dois estavam utilizando medicamentos diferentes de mesma classe terapêutica. Além disso, dois pacientes apresentavam efeitos colaterais, 14 estavam com dificuldades em encontrar os medicamentos prescritos e nove apresentavam dificuldades com o preço dos medicamentos. E em estudo realizado por Smith e colaboradores (1997), em que 53 idosos receberam visita domiciliar sete a dez dias após alta, verificou-se que 31 pacientes apresentaram alteração no esquema farmacoterapêutico prescrito na alta hospitalar. A intervenção, após contato com o médico prescritor, possibilitou prevenir sete readmissões hospitalares.

Vários estudos clínicos avaliaram o impacto de estratégias utilizadas para o gerenciamento da segurança dos pacientes após alta hospitalar. Schnipper e colaboradores (2006) demonstraram que, após orientação farmacêutica e seguimento por telefone de três a cinco dias após alta, o grupo sob intervenção apresentou menor número de eventos adversos relacionados a medicamentos que o grupo controle ( $1 \%$ versus $11 \%)$ e menor número de atendimentos em serviços de urgência ( $1 \%$ versus $8 \%)$.

Estudo em que a transição de pacientes idosos foi acompanhada por enfermeira, cujas funções incluíram reconciliação medicamentosa, orientação e capacitação do paciente, além de visitas domiciliares e contatos telefônicos, demonstrou que os pacientes sob intervenção tiveram menores taxas de 
readmissão hospitalar quando comparados ao grupo controle, aos 30, 90 e 180 dias após alta (COLEMAN et al., 2006).

Em dois estudos realizados com idosos, enfermeiras especializadas em gerontologia elaboraram plano de alta especializado, realizaram visitas domiciliares e contato telefônico semanal com o paciente ou seus familiares, e disponibilizaram um número de telefone durante sete dias por semana para esclarecimentos de dúvidas. No estudo realizado por Huang e Liang (2005), os resultados do grupo sob intervenção em comparação com o grupo controle foram: número de readmissão hospitalar após três meses (4 versus 13), mortes após alta (zero versus 4), melhor habilidade para realização de atividades diárias e maior pontuação em testes de avaliação da qualidade de vida. No outro estudo, os pacientes que receberam intervenção durante quatro meses após alta apresentaram, após um ano, menor taxa de readmissão hospitalar $(20 \%$ versus $37 \%)$, menor taxa de readmissōes múltiplas ( $6,2 \%$ versus $14,5 \%)$, menor número de casos de readmissóes relacionadas à admissão hospitalar no início do estudo (30 versus 64). O custo total no atendimento do grupo controle foi maior que o custo do grupo que recebeu intervenção - cerca de USD\$ 1.200 .000 versus USD\$ 600.000 (NAYLOR et al., 1999).

O conjunto de atividades desenvolvidas pela equipe hospitalar é essencial para o fornecimento de cuidado diferenciado na alta hospitalar, visando à segurança do paciente. Além disso, é necessário haver colaboração entre as instituições de saúde para que as transições de cuidado sejam efetivas (COLEMAN; BERENSON, 2004), uma vez que nenhum dos níveis de atendimento possui todos os recursos e competências necessários para resolver as necessidades de saúde de uma população (GIOVANELLA et al., 2009). No entanto, as instituições geralmente funcionam de forma isolada, e a fragmentação e desarticulação do cuidado contribuem para a perda da efetividade, aumento da iatrogenia e dos custos dos serviços de saúde e diluição da responsabilidade sanitária sobre os resultados dos atendimentos realizados (CAMPOS; DOMITTI, 2007).

\section{Articulação entre hospital, atenção básica e domicílio}

A integralidade do cuidado requer abordagem integral do indivíduo, articulação de ações de promoção, prevenção, cura e recuperação, e garantia da atenção nos três níveis de complexidade da assistência médica - atenção básica, secundária 
e hospitalar (CAMPOS, 2003). Embora essencial para a integração das redes

de saúde, a existência de um efetivo sistema de referência e contrarreferência não é suficiente para garantir continuidade do cuidado. É necessário haver responsabilização do profissional e do sistema pela saúde do usuário e, segundo CECILIO e MERHY (2003, p. 6):

O momento de alta de cada paciente deve ser pensado como um momento privilegiado para se produzir a continuidade do tratamento em outros serviços, não apenas de forma burocrática, cumprindo um papel da contra-referência, mas pela construção ativa da linha de cuidado necessária àquele paciente específico.

A linha de cuidado deve ter início na entrada do usuário em qualquer ponto do sistema que opere a assistência - Unidade Básica de Saúde (UBS), Unidade de Saúde da Família (USF), serviço de urgência - prosseguindo por serviços de apoio diagnóstico e terapêutico, especialidades, atenção hospitalar e outros, com acompanhamento da equipe da atenção básica durante todo o percurso, de forma a garantir fluxo seguro a todos os serviços que atendam às necessidades dos usuários, evitando interrupção e segmentação do cuidado, de forma a proporcionar cuidado integral (MALTA; MERHY, 2010; FRANCO; MAGALHÃES JÚNIOR, 2004). Para Malta e Merhy (2010, p. 597), “cada serviço pode ser repensado como um componente fundamental da integralidade do cuidado, como uma 'estação' no circuito que cada indivíduo percorre para obter a integralidade de que necessita” e, além de acesso e resolutividade, é necessário conectar esses serviços.

Para evitar a descontinuidade do atendimento e garantir a efetividade e segurança do tratamento, é essencial que o desenvolvimento das estratégias ocorra de forma articulada entre a equipe hospitalar e a equipe da atenção básica. Kripalani et al. (2007a) recomendam realização de contato para o compartilhamento de informações sobre o paciente, esclarecimento sobre ocorrências durante a hospitalização e para formulação de plano de seguimento do paciente.

Além do seguimento ambulatorial de pacientes após alta hospitalar, iniciativas como a Estratégia Saúde da Família, o programa federal "Melhor em Casa" e serviços de atendimento domiciliar oferecidos por convênios de saúde podem contribuir para evitar a descontinuidade do atendimento ao paciente após alta, especialmente quando integrados ao atendimento hospitalar. 
O processo de uso de medicamentos após alta hospitalar, com foco na segurança do paciente, é complexo e envolve múltiplas ações, além de atuação interdisciplinar e interinstitucional. A articulação entre os serviços de saúde pode possibilitar o desenvolvimento de uma linha de cuidado para a segurança do paciente no processo de uso de medicamentos, contribuindo para a integralidade do cuidado.

Conclui-se que o desenvolvimento de atividades de cuidado ao paciente em alta, pela equipe hospitalar, e a existência de efetiva articulação entre os diferentes níveis de complexidade do atendimento em saúde podem representar importantes aliados neste grande desafio para a saúde pública, que é a segurança do paciente no processo de uso de medicamentos após alta hospitalar. ${ }^{7}$

\section{Referências}

ANDREAZZA, R. S. et al. Causes of drug-related problems in the emergency room of a hospital in southern Brazil. Gaceta Sanitaria, v. 25, n.6, p. 501-506, 2011.

ARONSON, J.K.; FERNER, R.E. Clarification of Terminology in Drug Safety. Drug Safety, v. 28, n. 10, p. 851-870, 2005.

AMERICAN SOCIETY OF HEALTH. System Pharmacists. ASHP guidelines on pharmacist-conducted patient education and counseling. American Journal of Health-System Pharmacy, v. 54, p. 431-434, 1997.

BARNSTEINER, J.H. Medication Reconciliation. In: HUGUES, R. E. (Ed.). Patient safety and quality: an evidence-based handbook for nurses. Rockville: Agency for Healthcare Research and Quality, 2008. Disponível em: <http://www.ahrq.gov/qual/nurseshdbk/ docs/BarnsteinerJ_MR.pdf>. Acesso em: 20 nov 2012.

. Medication reconciliation: transfer of medication information across settings keeping it free from error. American Journal of Nursing, suppl., p. 31-36, 2005.

BAYLEY, K.B. et al. Evaluation of patient care interventions and recommendations by a transitional care pharmacist. Therapeutics and Clinical Risk Management, v. 3, n. 4, p. $695-$ 703, 2007.

BERGKVIST, A. et al. Improved quality in the hospital discharge summary reduces medication errors-LIMM: Landskrona Integrated Medicines Management. European Journal of Clinical Pharmacology, v. 65, p. 1.037-1.046, 2009.

BOOCKVAR, K. et al. Adverse events due to discontinuations in drug use and dose changes in patients transferred between acute and long-term care facilities. Archives of Internal Medicine, v. 164, p. 545-550, 2004. 
BRASIL. Portaria MS no 529, de $1^{\circ}$ de abril de 2013. Institui o Programa Nacional de Segurança do Paciente (PNSP). Diário Oficial da União, Brasília, DF.

BISOGNANO, M.; BOUTWELL, A. Improving transitions to reduce readmissions. Frontiers of Healthcare Services Management, v. 25, 2009. Disponível em: <http://www.ache. org/pdf/secure/gifts/July10-frontiers.pdf>. Acesso em: 20 nov 2011.

CALABRESE, A.T. et al. Pharmacist involvement in a multidisciplinary inpatient medication education program. American Journal of Health-System Pharmacy, v. 60, p. 1.012-1.018, 2003.

CAMPOS, C.E.A. O desafio da integralidade segundo as perspectivas da vigilância da saúde e da saúde da família. Ciência \& Saúde Coletiva. Rio de Janeiro, v. 8, n. 2, p. 569-584, 2003.

CAMPOS, G.W.S.; DOMITTI, A.C. Apoio matricial e equipe de referência: uma metodologia para gestão do trabalho interdisciplinar em saúde. Cadernos de Saúde Pública, Rio de Janeiro, v. 23, n. 2, p. 399-407, 2007.

CANO, F.G; ROZENFELD, S. Adverse drug events in hospitals: a systematic review. Cadernos de Saúde Pública, Rio de Janeiro, v. 25, supl. 3, p. S360-S372, 2009.

CECILIO, L.C.O.; MERHY, E.E. A integralidade do cuidado como eixo da gestão hospitalar, 2003. Disponível em: <http://www.uff.br/saudecoletiva/professores/merhy/capitulos-07. pdf>. Acesso em: 10 nov 2011.

CHEN, D.; BURNS, A. Summary and Recommendations of ASHP-APhA Medication Reconciliation Initiative Workgroup Meeting, 2007. Disponível em: <http://www.ashp. org/s_ashp/docs/files/MedRec_ASHP_APhA_Wkgrp_MtgSummary.pdf>. Acesso em: 03 ago 2012.

COLEMAN, E.A. et al. The care transitions intervention: results of a randomized controlled trial. Archives of Internal Medicine, v. 166, p. 1.822-1.828, 2006.

COLEMAN, E.A.; BERENSON, R.A. Lost in transition: challenges and opportunities for improving the quality of transitional care. Annals of Internal Medicine, v.140, p. 533536, 2004.

CORNISH, P.L. et al. Unintended medication discrepancies at the time of hospital admission. Archives of Internal Medicine, v.165, p. 424-429, 2005.

CUA, Y.M.; KRIPALANI, S. Medication use in the transition from hospital to home. Annals of Academy of Medicine Singapore, v. 37, p. 136-141, 2008.

EDWARDS, I.R.; JEFFREY, K.; ARONSON, J.K. Adverse drug reactions: definitions, diagnosis, and management. Lancet, v. 356, p. 1255-59, 2000.

FOSTER, A.J. et al. Adverse drug events occurring following hospital discharge. Journal of General Internal Medicine, v. 20, p. 317-323, 2005. 
Adverse events among medical patients after discharge from hospital. Canadian Medical Association Journal, v. 170, n. 3, p. 345-349, 2004.

The incidence and severity of adverse events affecting patients after discharge from the hospital. Annals of Internal Medicine, v. 138, p. 161-167, 2003.

FRANCO, T.B.; MAGALHÂES JÚNIOR, H.M. Integralidade na assistência à saúde: a organização das linhas do cuidado, 2004. Disponível em: <http://www.hc.ufmg.br/gids/ anexos/integralidade_assistencia_saude.pdf>. Acesso em: 20 nov 2012.

GIOVANELLA, L. et al. Saúde da família: limites e possibilidades para uma abordagem integral de atenção primária à saúde no Brasil. Ciência \& Saúde Coletiva. Rio de Janeiro, v.14, n.3, p.783-794, 2009.

GLEASON, K.M. et al. Reconciliation of discrepancies in medication histories and admission orders of newly hospitalized patients. American Journal of Health-System Pharmacy, v. 61, p. 1689-1695, 2004.

GLINTBORG, B.; ANDERSEN, S.E.; DALHOFF, K. Insufficient communication about medication use at the interface between hospital and primary care. Quality and Safety Health Care, v. 16, p. 34-39, 2007.

HOLLOWAY, A. Patient knowledge and information concerning medication on discharge from hospital. Journal of Advanced Nursing, v. 24, p. 1.169-1.174, 1996.

HUANG, T-T.; LIANG, S-H. A randomized clinical trial of the effectiveness of a discharge planning intervention in hospitalized elders with hip fracture due falling. Journal of Clinical Nursing, v. 14, p. 1.193-1.201, 2005.

KABOLI, P.J. et al. Clinical pharmacists and inpatient medical care - a systematic review. Archives of Internal Medicine, v.166, p. 955-963, 2006.

KERZMAN, H.; BARON-EPEL, O.; TOREN, O. What do discharged patients know about their medication? Patient Education and Counseling, v. 56, p. 276-282, 2005.

KOHN, L.T.; CORRIGAN, J.M.; DONALDSON, M.S. To err is human: building a safer health system. Washington, DC: National Academy Press, 2000.

KONGKAEW, C.; NOYCE, P.R.; ASHCROFF, D.M. Hospital admissions associated with adverse drug reactions: a systematic review of prospective observational studies. The Annals of Pharmacotherapy, v. 42, n. 7/8, p. 1.017-1.025, 2008.

KRIPALANI, S. et al. Deficits in communication and information transfer between hospital-based and primary care physicians implications for patient safety and continuity of care. Journal of the American Medical Association, v. 297, p. 831-841, 2007 b.

. Promoting effective transitions of care at hospital discharge: a review of key issues for hospitalists. Journal of Hospital Medicine, v. 2, n. 5, p. 314-322, 2007a. 
MALTA, D.C.; MERHY, E.E. O percurso da linha do cuidado sob a perspectiva das doenças crônicas não transmissíveis. Interface - Comunicação, Saúde, Educação. Botucatu, v. 14, n. 34, p. 593-605, 2010.

MAMON, J. et al. Impact of hospital discharge planning on meeting patient needs after returning home. Health Services Research, v. 27, n. 2, p. 155-174, 1992.

MENDES, W. et al. Revisão dos estudos de avaliação da ocorrência de eventos adversos em hospitais. Revista Brasileira de Epidemiology, v. 8, n. 4, p. 393-406, 2005.

. The assessment of adverse events in hospitals in Brazil. International Journal for Quality in Health Care, v. 21, n. 4, p. 279-284, 2009.

MISTIAEN, P.; FRANCKE, A.L.; POOT, E. Interventions aimed at reducing problems in adult patients discharged from hospital to home: a systematic meta-review. BioMed Central Health Services Research, v.7, 2007. Disponível em: < http://www.biomedcentral.com/14726963/7/47. Acesso em: 18 nov 2012.

MORIEL, M.C. et al. Prospective study on conciliation of medication in orthopaedic patients. Farmacia Hospitalaria, v. 32, n. 2, p. 65-70, 2008.

MUELLER, S.K. et al. Hospital-based medication reconciliation practices: a systematic review. Archives of Internal Medicine. Eletronico 2012. Disponível em: <http://archinte. jamanetwork.com>. Acesso em: 26 jun 2012.

NAYLOR, M.D. et al. Comprehensive discharge planning and home follow-up of hospitalized elders: a randomized clinical trial. Journal of the American Medical Association, v. 281, n. 7, p. 613-620, 1999.

NOBLAT, A.C. B. et al. Prevalência de admissão hospitalar por reação adversa a medicamentos em Salvador, BA. Revista da Associação Médica Brasileira, São Paulo, v. 57, n. 1, p. 42-45, 2011.

NORMAN, A.H.; TESSER, C.D. Prevenção quaternária na atenção primária à saúde: uma necessidade do Sistema Único de Saúde. Cadernos de Saúde Pública. Rio de Janeiro, v. 25, n. 9, p. 2012-2020, 2009.

OLIVEIRA, C.C.; REIS, A. Questões epistemológicas e bioéticas da prevenção quaternária. Physis: Revista de Saúde Coletiva, v. 22, n. 4, p. 1.485-1.502, 2012.

PFAFFENBACH, G.; CARVALHO, O.M.; BERGSTEN-MENDES, G. Reações adversas a medicamentos como determinantes da admissão hospitalar. Revista da Associação Médica Brasileira, São Paulo, v. 48, n. 3, p. 237-241, 2002.

PIPPINS, J.R. et al. Classifying and predicting errors of inpatient medication reconciliation. Journal of General Internal Medicine, v. 23, n. 9, p. 1.414-1.419, 2008.

PIRMOHAMED, M. et al. Adverse drug reactions as cause of admission to hospital: prospective analysis of 18820 patients. British Medical Journal, v. 329, p. 15-19, 2004. 
RANTUCCI, M.J. Pharmacists talking with patients: a guide to patient counseling. $2^{\text {nd }}$ ed. Baltimore: Lippincott Williams \& Wilkins, 2007.

RATLIFF, B.W. Leaving the hospital: discharge planning for total patient care. Springfield: Charles C. Thomas, 1981.

ROMANO, C.A. Computerized multidisciplinary discharge care planning. Proceedings of the Annual Symposium Computer Application in Medical Care, v. 2, p. 587-589, 1982.

ROZENFELD, S. Agravos provocados por medicamentos em hospitais do Estado do Rio de Janeiro, Brasil. Revista de Saúde Pública, São Paulo, p. 1-8, 2007.

RUNCIMAN, W. et al. Towards an international classification for patient safety: key concepts and terms. International Journal for Quality in Health Care, v. 21, n. 1, p. 18-26, 2009.

SCHNIPPER, J.L. et al. Role of pharmacist counseling in preventing adverse drug events after hospitalization. Archives of Internal Medicine, v. 166, p. 565-571, 2006.

SILVA, T.; SCHENKEL, E.P.; MENGUE, S.S. Nível de informação a respeito de medicamentos prescritos a pacientes ambulatoriais de hospital universitário. Cadernos de Saúde Pública, v. 16, n. 2, p. 449-455, 2000.

SMITH, L. et al. An investigation of hospital generated pharmaceutical care when patients are discharged home from hospital. British Journal of Clinical Pharmacology, v. 44, p. 163165, 1997.

STUFFKEN, R.; EGBERTS, T.C.G. Discontinuities in drug use upon hospital discharge. Pharmacy World \& Science, v. 26, p. 268-270, 2004.

TACHÈ, S.V.; SÖNNICHSEN, A.; ASHCROFT, D.M. Prevalence of adverse drug events in ambulatory care: a systematic review. Annals of Pharmacotherapy, v. 45, n. 7/8, p. 977989, 2011.

UNROE, K.T. et al. Inpatient medication reconciliation at admission and discharge: a retrospective cohort study of age and other risk factors for medication discrepancies. The American Journal of Geriatric Pharmacotherapy, v. 8, p. 115-126, 2010.

VAN WALRAVEN, C. et al. Effect of discharge summary availability during post-discharge visits on hospital readmission. Journal of General Internal Medicine, v. 17, p. 186-192, 2002.

VARALLO, F.R. Internaçôes hospitalares por reações adversas a medicamentos (RAM) em um hospital de ensino. 2010. 96 f. Dissertação (Mestrado). Faculdade de Ciências Farmacêuticas, Universidade Paulista “Júlio de Mesquita Filho”, Araraquara, 2010.

VIRA, T.; COLQUHOUN, M.; ETCHELLS, E. Reconciliable differences: correcting medication errors at hospital admission and discharge. Quality and Safety in Health Care, v. 15, p.122-126, 2006.

VRIES, E.N. et al. The incidence and nature of in-hospital adverse events: a systematic review. Quality and Safety in Health Care, v.17, p.216-223, 2008. 
WACHTER, R.M. Compreendendo a segurança do paciente. Porto Alegre: Artmed, 2010.

WILliANS, S. What is the High 5s Project? High 5's Project Website. Disponível em: https://www.high5s.org/bin/view/Main/WebHome. Acesso em 19 nov 2012.

WORLD HEALTH ORGANIZATION. International drug monitoring: the role of national centers. Geneva: World Health Organization, 1972.

\section{Notas}

${ }^{1}$ Informação extraída dos arquivos da World Health Organization. Disponível em: http://www.who. int/patientsafety/worldalliance/alliance/en/index.html. Acesso em: 07 abr. 2013.

${ }^{2}$ Evento adverso (EA) é "um incidente que resulta em dano ao paciente" (RUNCIMAN et al., 2009, p. 23).

${ }^{3}$ Evento adverso relacionado a medicamento (EAM) é "qualquer ocorrência indesejável que pode se apresentar durante tratamento com um produto farmacêutico, mas que não tenha, necessariamente, relação causal com o tratamento" (EDWARDS; ARONSON, 2000, p. 1256). Os danos ao paciente podem ser produzidos por diversos mecanismos, tais como reação adversa a medicamento, erros de medicação (prescrição, dispensação ou administração de medicamentos) e uso de medicamentos com desvio de qualidade.

${ }^{4}$ Reação adversa a medicamento (RAM) foi definida pela Organização Mundial da Saúde (WHO, 1972, p. 9) como "resposta a um medicamento que é nociva, não intencional e que ocorre em doses normalmente utilizadas no homem para profilaxia, diagnóstico, tratamento ou modificação de função fisiológica". Excluem-se, portanto, as superdosagens acidentais ou intencionais. Edwards e Aronson (2000, p. 1.255) definem RAM como "uma reação consideravelmente nociva ou desagradável, resultante de uma intervenção relacionada com o uso de um medicamento, que prediz perigo de futura administração e justifica prevenção, tratamento específico, alteração de dose ou retirada do produto". Recentemente, foi proposta uma nova definição para RAM, referindo-se a "dano inesperado resultante de uma ação justificada, onde o processo correto foi seguido para o contexto no qual o evento ocorreu" (RUNCIMAN et al., 2009, p. 21). Entretanto, os estudos aqui apresentados não fizeram distinção entre os danos inesperados e os danos decorrentes de efeitos colaterais, considerados desfechos previsíveis, uma vez que estão relacionados às propriedades farmacológicas do medicamento, ou, ainda, outros danos decorrentes de efeitos já conhecidos e documentados.

${ }^{5}$ Os termos efeito adverso e reação adversa se referem ao mesmo fenômeno, sendo o primeiro a partir do ponto de vista do medicamento, e o segundo, a partir do ponto de vista do paciente: um medicamento causa um efeito, enquanto que o paciente tem uma reação (ARONSON; FERNER, 2005).

${ }^{6}$ Erro é uma "falha em realizar uma ação planejada ou aplicação de um plano incorreto" (RUNCIMAN et al., 2009, p. 22). Os danos decorrentes de erros de medicação são considerados eventos adversos evitáveis (WACHTER, 2010).

${ }^{7}$ L.F.G. Marques contribuiu com a concepção, pesquisa bibliográfica, análise de dados e redação do artigo. N.S. Romano-Lieber contribuiu com a concepção, redação e revisão crítica do artigo. 
Patient safety strategies for safe use of
medications after hospital discharge

The current global movement for patient safety has

focused on quaternary prevention - actions to avoid

excessive diagnostic and therapeutic interventions and to minimize the risk of iatrogenicity - to prevent harm to patients and unnecessary costs to the health system. In transitional care situations such as hospital discharge the occurrence of adverse drug events may require hospital readmission or outpatient or emergency care. This article aimed to provide input to the discussion on patient safety management for medication use after hospital discharge. A literature search was conducted and strategies such as medication reconciliation, patient and/or caregiver counseling, discharge summary communication and follow-up after discharge are addressed. In addition, it is stressed the need for interdisciplinary and interinstitutional actions for comprehensive care. In conclusion, patient care provided by hospital teams and effective coordination of care by the hospital and other health services are crucial to meet this significant public health challenge of ensuring patient safety on medication use after discharge.

> Key words: medication, adverse events, hospital, patient safety, public health. 